\title{
Selection of Bit-rate for Wireless Network Coding
}

\author{
Bin Ni, Naveen Santhapuri, Chase Gray, Srihari Nelakuditi \\ Department of Computer Science and Engineering \\ University of South Carolina \\ Columbia, SC 29205 \\ Email: \{nib,santhapu,graycm,srihari\}@cse.sc.edu
}

\begin{abstract}
Network coding is known to improve throughput by mixing information from different flows and conveying more information in each transmission. Recently some proposals have demonstrated the benefits of applying network coding to wireless networks with broadcast transmissions. It is expected that the opportunities for coding and the corresponding gains depend on the bit-rate chosen for determining routes and transmitting packets. However, the previous work on wireless network coding assumed a fixed rate and did not explicitly account for the interaction between rate selection and coding gain. In this paper, we define a new metric, expected coded time (ECT), that measures the total time needed by a node to deliver two packets to their receivers given the bit-rate for transmitting coded packets. We then investigate how the optimal bit-rate for coded packets differs from that for transmission of native packets individually. We also study the performance of network coding under different fixed bit-rates for the whole network. Our evaluation shows that 11 Mbps is the best default fixed rate for MIT Roofnet and $5.5 \mathrm{Mbps}$ is mostly the optimal rate to transmit coded packets when the ideal individual bit-rate for each receiver is different.
\end{abstract}

\section{INTRODUCTION AND MOTIVATION}

Network coding is a way of mixing data at intermediate nodes for efficient communication with some additional computation. It is one of the few options available to stretch the capacity of existing wireless technologies. A simple example of network coding in a wireless context is shown in Figure 1, where two nodes $n 1$ and $n 2$ exchange packets $a$ and $b$ via router $R$. Without coding, this exchange requires 4 transmissions. On the other hand, after receiving both $a$ and $b$, node $R$ can broadcast the coded packet $a \oplus b$. The two nodes $n 1$ and $n 2$ can decode their respective packets using the buffered packet and the coded packet. Thus, with coding, the exchange needs only 3 instead of 4 transmissions.

A practical scheme, referred to as COPE, based on network coding for wireless networks is proposed in [8]. COPE extends the gain of coding beyond the above information exchange scenario through opportunistic coding of two or more packets in a single transmission. COPE does this by having nodes overhear others transmissions in their neighborhood and issue reception reports to let neighbors learn about the packets they currently have. It is reported that COPE with its opportunistic listening and coding achieves several fold increase in the network throughput.

The operations under COPE are independent of the bitrate chosen for determining routes and transmitting packets. Actually, the experiments described in [8] are based on the

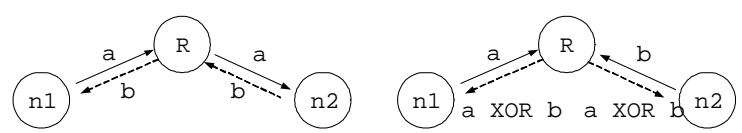

Fig. 1. An exchange example
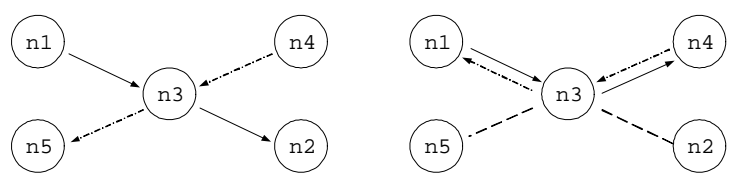

Fig. 2. Illustration of the effect of bit-rate on routing and coding

fixed bit-rate of $6 \mathrm{Mbps}$ in an 802.11a network. They do not consider the interaction between different fixed bit-rates and the resulting coding opportunities. Consider the example illustrated in Figure 2. Suppose $n 5$ and $n 2$ can not overhear $n 1$ and $n 4$, and two flows exist from $n 1$ to $n 2, n 4$ to $n 5$ (source and destinations for both flows are not shown here). In this scenario, COPE cannot achieve any coding gain. However, suppose a rate change causes the topology to be changed to the one on the right, i.e., the flow through $n 4-n 3-n 5$ changes its route to $n 4-n 3-n 1$ and the flow through $n 1-n 3-n 2$ changes its route to $n 1-n 3-n 4$. In this scenario, the entire traffic is concentrated among the three nodes $n 1, n 3, n 4$ and network coding can be applied directly for such an exchange. We elaborate further on this interaction below.

If the sender transmits at a lower bit-rate, then its packet is likely heard by some far away nodes due to low SINR (signal to interference and noise ratio) requirements at lower rates. Each node will have many neighbors which is likely to increase coding opportunities. On the other hand, the resulting routes would have few long-hops and not many routes may pass through a given node, limiting the opportunities for coding. Conversely, if the sender transmits at a higher bitrate, the packet loss probability would be high and the number of neighbors might be few, with less coding opportunities. But due to routes with many short-hops, several routes may pass through a given node, and so chances for coding may be high. Therefore, it is clear that bit-rate selection impacts the performance of a coding scheme such as COPE. However, it is not apparent whether coding opportunities increase or decrease with higher bit-rates. More importantly, which rate gains more throughput from these opportunities is not obvious. 
This paper focuses on the interplay between bit-rate selection and coding gain. We consider both fixed rate selection for the whole network, and dynamic rate selection for each coded transmission. We define a new metric, expected coded time (ECT), that measures the total time needed by a node to deliver two packets to their receivers given the bit-rate for transmitting coded packets. We then investigate how the optimal rate for coded packets differs from that for transmission of individual packets. We also study the performance of network coding under different fixed rates for the whole network.

The organization of this paper is as follows. The related work is discussed in the next section. The new metric ECT is introduced in Section III. The evaluation of coding gain at different fixed and dynamic bit-rates for MIT Roofnet is presented in Section IV. We then conclude the paper with discussion on future work in Section V.

\section{RELATED WORK}

A comprehensive description of the theoretical foundations of network coding is given in [1]. COPE [8] is one of the first schemes in which the practical viability of wireless network coding was demonstrated. One of the recent works on wireless network coding, apart from COPE, is a new opportunistic routing protocol called MORE [3]. In MORE, the routers code packets going to the same destination and forward the coded packets. The destination decodes and recovers the original packets. This approach needs no coordination and maximizes network throughput. While MORE performs intra-flow coding, the focus of this work is on inter-flow coding schemes such as COPE. There have been some proposals to create more coding opportunities with coding-aware routing [11], [12]. Since bitrate chosen for the whole network impacts the routes packets traverse, this work does somewhat indirectly studies the effect of routing on coding opportunities and gains. Recently, a new way of network coding has been proposed which encodes signals instead of bits [7], [13]. While this work is about encoding bits, with dynamic bit-rate selection, in some sense, it does control the physical layer transmission of signals.

There are many schemes proposed for selecting bit-rate at the MAC layer [2], [5], [6], [9]. Auto Rate Fallback (ARF) is the original bit-rate selection algorithm created for the WaveLAN-II 802.11 cards [6] Adaptive Auto Rate Fallback (AARF) [9] is an extension of ARF where step-up parameter is doubled every time the algorithm tries to increase the bit-rate and the subsequent packet fails. The MadWifi device driver for Atheros cards uses the Onoe algorithm which is much less sensitive to individual packet failure than ARF. Receiver Based Auto-Rate (RBAR) [5] chooses the bit-rate based on $\mathrm{S} / \mathrm{N}$ measurements at the receiver. SampleRate [2] deals with bit-rate selection based on estimates of the expected per-packet transmission time at each bit-rate. All of these rate selection schemes are interested only in determining the best rate for a single receiver. Our work in this paper focuses on selecting the optimal rate for transmitting a coded packet to two receivers.
TABLE I

EFFECTIVE DATA TRANSMISSION RATES (IN MBPS) FOR 802.11B FOR DIFFERENT PACKET SIZES

\begin{tabular}{c||c|c|c} 
Rate & 4000 bits & 8000 bits & 12000 bits \\
\hline 1 & 0.9542 & 0.97656 & 0.98425 \\
2 & 1.82482 & 1.90840 & 1.93798 \\
5.5 & 4.35127 & 4.85866 & 5.05515 \\
11 & 7.19895 & 8.70253 & 9.35374
\end{tabular}

\section{Rate Selection For CODEd Transmissions}

In this section, we first discuss a previously proposed routing metric Expected Transmission Time (ETT) and then define a new metric, Expected Coded Time (ECT) for delivering two packets with coding. ECT helps in making the rate selection for transmitting a coded packet to two receivers. We then use an example to illustrate how different rate choices compare with the optimal rate for coded transmissions.

\section{A. Expected Transmission Time}

Expected Transmission Count (ETX) metric was proposed in [4] to model the expected number of transmissions required to send a unicast packet over a link, including retransmissions. To calculate ETX, each node measures the probability that a packet successfully reaches the receiver, denoted as $d_{f}$, and the probability that an ACK is successfully received by the sender, denoted as $d_{r}$. The ETX value of the link is then given by $\frac{1}{d_{f} \cdot d_{r}}$. To account for different transmission rates, another metric Estimated Transmission Time (ETT) was proposed, which is estimated as ETT $=\mathrm{ETX} \cdot \frac{S}{r}$, where $S$ is the size of the packet and $r$ is the "effective" data transmission rate. Note that physical layer preamble time (192 $\mu$ s for $802.11 \mathrm{~b})$ must be taken into account when determining the effective rate and these rates for $802.11 \mathrm{~b}$ for varying packet sizes can be seen in Table I.

\section{B. Expected Coded Time}

The ETT captures the time to deliver a packet from a sender to one receiver. However, with network coding, the coded packet is broadcast by a sender to at least two receivers each with different delivery probabilities. There is no existing metric that captures the time taken to deliver packets with coding. In this section, we define a new metric, Expected Coded Time (ECT), for exchanging two packets between two neighbors of a node with coded transmissions as in Fig. 1.

Suppose the transmission rate for broadcasting coded packets is $r$. Let $r_{1}^{*}$ be the optimal transmission rate for a unicast transmission between $R$ and $n_{1}$ that yields the lowest ETT. Similarly $r_{2}^{*}$ be the optimal rate between $R$ and $n_{2}$. Also, assume that the corresponding successful delivery ratios are $p_{1}$ and $p_{2}$ respectively. We denote the loss probabilities as $s_{1}=1-p_{1}$ and $s_{2}=1-p_{2}$. If one of the neighbors received (say $\mathrm{n} 1$ ) the coded packet, the router $\mathrm{R}$ need not broadcast the coded packet at the rate $r$ until the other node (say n2) receives it. Instead, $\mathrm{R}$ can transmit the coded packet to the node $\mathrm{n} 2$, that has not yet received it using the optimal rate for $\mathrm{n} 2\left(r_{2}^{*}\right)$. This will improve the performance by transmitting 
at the optimal rate as much as possible. The new metric ECT that gives the time for exchanging two packets between two neighbors of an intermediate node with optimal individual rates $r_{1}^{*}$ and $r_{2}^{*}$ when the coded transmission is broadcast at rate $r$ can then be expressed as:

$$
\begin{array}{r}
E C T\left(r, r_{1}^{*}, r_{2}^{*}\right)= \\
\frac{S}{r} \sum_{i=1}^{\infty} s_{1}^{i-1} p_{1} s_{2}^{i} i \\
+\frac{S}{r_{2}^{*}} \sum_{i=1}^{\infty} s_{1}^{i-1} p_{1} s_{2}^{i} \sum_{j=i+1}^{\infty}(j-i)\left(s_{2}^{r_{2}^{*}}\right)^{j-i+1} p_{2}^{r_{2}^{*}} \\
+\frac{S}{r} \sum_{i=1}^{\infty} s_{2}^{i-1} p_{2} s_{1}^{i} i \\
+\frac{S}{r_{1}^{*}} \sum_{i=1}^{\infty} s_{2}^{i-1} p_{2} s_{1}^{i} \sum_{j=i+1}^{\infty}(j-i)\left(s_{1}^{r_{1}^{*}}\right)^{j-i+1} p_{1}^{r_{1}^{*}} \\
\left.+\frac{S}{r} \sum_{i=1}^{\infty} s_{1}^{i-1} s_{2}^{i-1} p_{1} p_{2} i\right]
\end{array}
$$

The result of Formula 1 is the expected time for $n_{1}$ to receive the coded packet first when $R$ broadcasts the coded packet using transmission rate $r$. Formula 2 captures the expected time for $R$ to transmit a coded packet with a unicast transmission to $n_{2}$ at the optimal transmission rate $r_{2}^{*}$ after $n_{1}$ has already acknowledged the coded packet. Formulas 3 and 4 are the same as Formulas 1 and 2, except they handle the case when $n_{2}$ receives the packet first. Finally, Formula 5 is the expected transmission time for $n_{1}$ and $n_{2}$ to receive the coded packet simultaneously using transmission rate $r$. Summing these sub-formulas together, we can obtain the expected transmission time for $R$ to deliver a coded packet to both neighbors, $n_{1}$ and $n_{2}$.

It can be reduced as:

$$
\frac{1}{p_{1}+p_{2}-p_{1} \cdot p_{2}} \cdot\left[\frac{S}{r}+\frac{S}{r_{2}^{*}} \cdot \frac{p_{1} \cdot s_{2}}{p_{2}^{r_{2}^{*}}}+\frac{S}{r_{1}^{*}} \cdot \frac{p_{2} \cdot s_{1}}{p_{1}^{r_{1}^{*}}}\right]
$$

If the same rate is optimal for both links, i.e., the value of $r, r_{1}^{*}$ and $r_{2}^{*}$ are the same, this can be simply reduced to the following formula:

$$
\operatorname{ECT}(r, r, r)=\left[\frac{1}{p_{1}}+\frac{1}{p_{2}}-\frac{1}{p_{1}+p_{2}-p_{1} p_{2}}\right] \cdot \frac{S}{r}
$$

which is equivalent to the Expected number of Coded Transmissions (ECX) scaled for rate, proposed in [11].

TABLE II

AN EXAMPLE WHEN MIN RATE IS OPTIMAL

\begin{tabular}{cccccc}
\hline \hline Rate & $p_{7,28}$ & $p_{7,22}$ & $E T T_{7,28}$ & $E T T_{7,22}$ & $E C T$ \\
\hline 1Mbps & 0.885 & 0.987 & 9319.122 & 8356.051 & 8402.784 \\
2Mbps & 0.947 & 0.693 & 4422.895 & 6043.985 & 4935.255 \\
5.5Mbps & 0.913 & 0.757 & 1791.886 & $2161.152^{*}$ & $2231.059^{*}$ \\
11Mbps & 0.885 & 0.368 & $1039.029^{*}$ & 2498.751 & 2342.543 \\
\hline
\end{tabular}

TABLE III

AN EXAMPLE WHEN MAX RATE IS OPTIMAL

\begin{tabular}{cccccc}
\hline \hline Rate & $p_{16,7}$ & $p_{16,32}$ & $E T T_{16,7}$ & $E T T_{16,32}$ & $E C T$ \\
\hline 1Mbps & 0.857 & 0.961 & 9623.597 & 8582.126 & 8496.309 \\
2Mbps & 0.856 & 0.745 & 4893.086 & 5622.123 & 4910.961 \\
5.5Mbps & 0.919 & 0.817 & 1780.187 & $2002.438^{*}$ & 2068.219 \\
11Mbps & 0.939 & 0.454 & $979.276^{*}$ & 2025.419 & $2041.285^{*}$ \\
\hline
\end{tabular}

TABLE IV

A CASE WHEN OPTIMAL RATE IS DIFFERENT FROM BOTH Min AND MAX

\begin{tabular}{cccccc}
\hline \hline Rate & $p_{1,24}$ & $p_{1,37}$ & $E T T_{1,24}$ & $E T T_{1,37}$ & $E C T$ \\
\hline 1Mbps & 0.987 & 0.949 & 8356.051 & 8690.646 & 8358.670 \\
2Mbps & 0.987 & 0.921 & 4243.649 & 4547.754 & 4349.852 \\
5.5Mbps & 0.997 & 0.872 & 1640.915 & 1876.137 & $1877.247^{*}$ \\
11Mbps & 0.96 & 0.493 & $957.854^{*}$ & $1865.193^{*}$ & 1884.473 \\
\hline
\end{tabular}

\section{Optimal Rate for Coded Transmissions}

Based on ECT metric, the optimal rate for coded transmissions is the rate that minimizes ECT. Let $r_{1}^{*}$ and $r_{2}^{*}$ be best rates for transmitting individual packets to each of the receivers. Then, obvious rate choices for transmitting coded packets are $\operatorname{Min}\left(r_{1}^{*}, r_{2}^{*}\right)$, the minimum among the optimal rates for each link individually and $\operatorname{Max}\left(r_{1}^{*}, r_{2}^{*}\right)$, the maximum rate among the two optimal rates. Tables II, III, and IV show the ETT and ECT values for each link in a coded exchange for Roofnet extracted scenarios. The values $p_{i, j}$ and $\mathrm{ETT}_{i, j}$ refer to the delivery probability and ETT for link $i \rightarrow j$ at the corresponding rate (shown in the 1st column). In Table II, link $7 \rightarrow 28$ has the least ETT value at $11 \mathrm{Mbps}$ and link $7 \rightarrow 22$ at 5.5 Mbps. The optimal data rate is $5.5 \mathrm{Mbps}$ which has the least ECT value. So, $\operatorname{Min}\left(r_{1}^{*}, r_{2}^{*}\right)$ is optimal for the exchange $22 \leftarrow 7 \rightarrow 28$. Similarly it can be observed that $\operatorname{Max}\left(r_{1}^{*}, r_{2}^{*}\right)$ is the optimal for the exchange $7 \leftarrow 16 \rightarrow 32$ in Table III. For the coded exchange $24 \leftarrow 1 \rightarrow 37$ shown in Table IV, the optimal rate for each of the links $(1 \rightarrow 24$ and $1 \rightarrow 37)$ is $11 \mathrm{Mbps}$ but the optimal rate for the coded packet is $5.5 \mathrm{Mbps}$ which has the least ECT value. It is interesting to see that there exist scenarios where each of the Min. rate and Max. rate are optimal and also scenarios where neither of them is optimal. Our evaluation in the next section investigates the optimal rates for coded transmissions in Roofnet. We also study how often the optimal rate matches Min or Max, or deviates from both.

\section{Evaluation}

For our evaluation we use the publicly available MIT Roofnet [10] trace data consisting of 38 nodes after excluding all unidirectional links and isolated nodes in the trace data. The data was originally obtained by monitoring the transmission of packets from each node for 90 seconds. All ETT and ECT values for each source and destination pair mentioned in this paper are calculated based on delivery ratios from this trace.

We evaluate with two different data sets. The first data set is based on the average delivery ratios over the entire trace for each link. For the second data set we extracted the delivery ratios for each link during 90 one second snapshots of the 


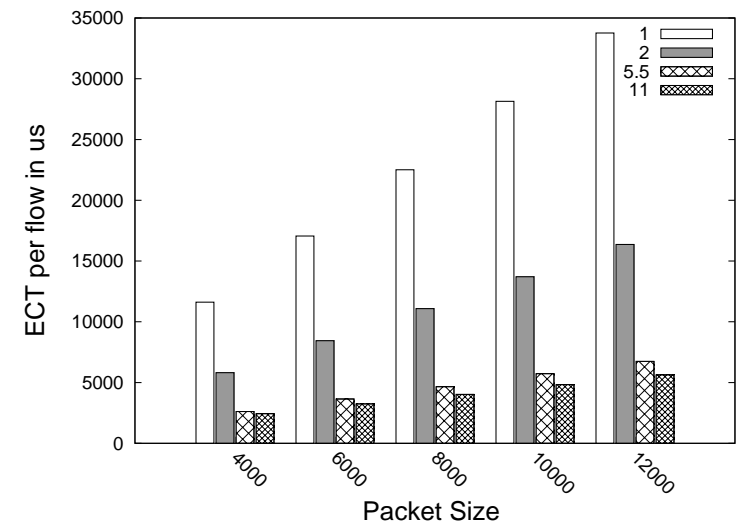

Fig. 3. ECT value per flow for different rates with different packet size

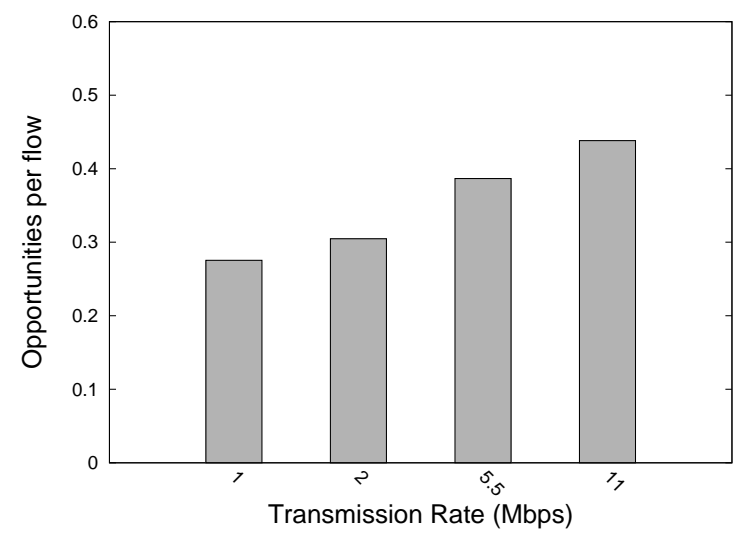

Fig. 4. Average coding opportunities per flow for different rates

network. We obtained this link information for each of the available $802.11 \mathrm{~b}$ transmissions rates: $1,2,5.5$, and $11 \mathrm{Mbps}$.

This paper brings up the following two important questions that we attempt to address with this evaluation.

1) When network coding is employed, which transmission rate is ideal for the network as a whole?

2) Given a coding opportunity at a node, which bit-rate should be used for broadcasting the coded packet?

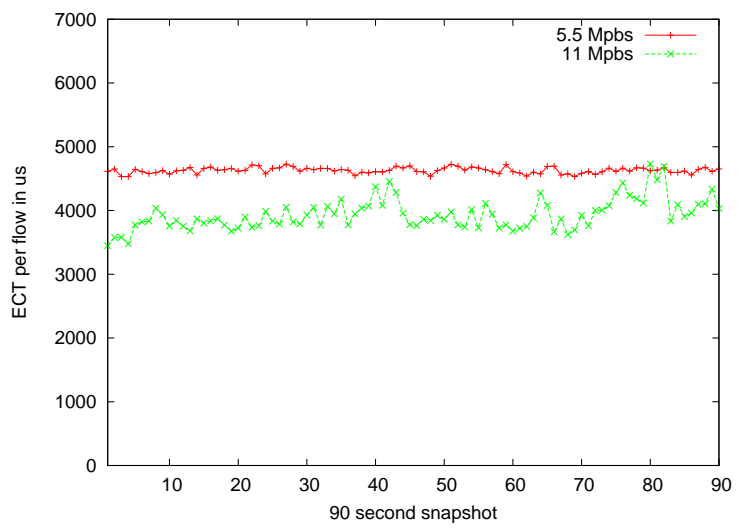

Fig. 5. ECT per flow with minimum hop count 2



Fig. 6. Total coding opportunities of exchange with minimum hop count 2

\section{A. Network-Wide Rate Selection}

In networks which operate on a fixed bit rate and use coding, the rate selection not only impacts the total time for transmissions but also impacts the route selection and as a result the number of coding opportunities. To investigate the impact of transmission rate on an entire network, we first found the shortest paths between all source and destination pairs in the Roofnet trace data separately for each of the four data rates, using ETT as the path metric. Due to varying link qualities at different transmission rates, the four route sets have a different set of links, thus forming different topologies. For each of these topologies we measured the coding opportunities and the average ECT value per flow. Below we show the results for the two data sets (90 second average and second-by-second snapshots). We considered only source destination node pairs with a minimum hop count of two to increase consistency between graphs and for clarity.

We extracted the opportunities for coding exchanges from all calculated shortest paths as follows: We obtained all node triples $n_{i} \rightarrow n_{j} \rightarrow n_{k}$ and $n_{k} \rightarrow n_{j} \rightarrow n_{i}$ which the respective shortest paths travel through. If the number of flows through each of the node triples is greater than 0, then there is opportunity for coding at the intersection. The number of packets that can be coded is equal to the minimum number of flows traveling through either of the triples. Using this value we can calculate the total transmission time for a coded exchange with the fixed rate ECT metric (Formula 7).

We calculated the total ECT value for all the coded exchanges for a given topology and obtained the ECT per flow value. Fig. 3 shows the ECT per flow for each data rate. We show a separate set of values for each packet size to show the effect of packet size on relative ECT values of different rates. From the figure we can see that $11 \mathrm{Mbps}$ will always offer the lowest ECT/flow in the Roofnet topology. To gauge the variation in coding opportunities at different data rates, we measured the average number of coding opportunities per flow. We considered only the typical packet size of 8000 bits for this evaluation. The results are summarized in Fig. 4 and show that $11 \mathrm{Mbps}$ has the most coding opportunities available 
compared to all other transmission rates.

We repeated the above two experiments for the second data set of 90 second snap shots. Fig. 5 shows the ECT per flow at each snap shot for 5.5 and $11 \mathrm{Mbps}$ (we omitted 1 and $2 \mathrm{Mbps}$ for the sake of clarity and also because they perform far worse than 5.5 and $11 \mathrm{Mbps}$ ). The results are similar to the 90 second average data set where $11 \mathrm{Mbps}$ offers the best performance. The cumulative coding opportunities during each snap shot are shown in Fig. 6 supporting the 11Mbps trend.

From these results we can conclude that $11 \mathrm{Mbps}$ is the best fixed global transmission rate for route selection in Roofnet if network coding is being used. The combination of lower ECT values and higher coding opportunities per flow will increase the performance of a fixed rate $11 \mathrm{Mbps}$ network more than other rates.

\section{B. Rate Selection for Coded Transmissions}

The previous section shows that by selecting appropriate rate for the whole network we can create more coding opportunities. Another interesting question then is when a coding opportunity arises at a node, which bit-rate should be used for broadcasting the coded packet? Take our first simple scenario of exchange in Fig. 1 as an example. The router has several strategies to send the coded packet. First, it may send the original packets using optimal individual rate for each link with unicast transmissions. Second, the router may broadcast the coded packet using the minimum or the maximum of the optimal rates of the two links. Third, the router may select an optimal rate for the coded packet which could be different from the optimal rates for each of the receivers.

We use the ECT metric described in the previous section for finding the best rate for coded transmissions. Recall that ECT is the time to deliver a coded packet to both the destinations (with the assumption that only 2 packets are coded together). The optimal rate can be determined by comparing the ECT vales and simple taking the rate which produces the least ECT value. To observe the difference in performance between the rate selection strategies (No coding+Dynamic rate, Min, Max), we plot the ECT values for the optimal rate vs the ETT/ECT values for the corresponding strategy in Fig. 7. This evaluation is performed on data set 1 (90 second average) with 8000 bit packets. Fig. 7(a) shows that a coding scheme with optimal routing can perform significantly better than a scheme without coding as most of the points in the scatter plot are skewed towards the high ETT values. Fig. 7(b) and Fig. 7(c) compare the optimal rate selection with Min. rate and Max. rate respectively. These figures show that Min consistently offers equivalent performance to the optimal rate, though some points deviate and Max deviates a lot from optimal rate.

To gain more insight into the rate selection we quantized the strategies that offer the best performance in Table $\mathrm{V}$ with different packet sizes on the 90-snapshot data set. This data shows that, Min and Max are equivalent approximately $90 \%$ of the time. Max is the optimal rate less than $0.4 \%$ of the time and Min is the optimal rate approximately $10 \%$ of the time when it is not equal to Max. There are some rare cases,



Fig. 8. Fraction of exchanges in which $11 \mathrm{Mbps}$ and 5.5 Mbps are chosen

less than $0.3 \%$, in which Min and Max are both not the optimal rate. We can conclude that using the minimum of the optimal rates of the two links will offer best performance over $99.4 \%$ of the time for different packet sizes. This is the sum of the amount of times the Min is the optimal rate and the amount of times Min and Max are both equal while also being the optimal rate. The $90 \%$ cases where Max and Min are equivalent might be the result of Roofnet topology and the few rates available in $802.11 \mathrm{~b}$. By using $802.11 \mathrm{a} / \mathrm{g}$ which have 8 data rates and/or by evaluating on a more denser topology, we expect this percentage to be a lot lower and result in a less skewered optimal rate distribution. For this reason, though selecting the lower rate among the two links for transmitting a coded packet seems intuitive (and is supported by our results), it may not guarantee the best ECT value in all cases.

TABLE V

PERCENTAGE OF OPTIMAL RATES FOR DIFFERENT PACKET SIZE

\begin{tabular}{ccccc}
\hline \hline & Max & Min & Both & Neither \\
\hline 4000 bits & $0.36 \%$ & $11.17 \%$ & $88.19 \%$ & $0.28 \%$ \\
8000 bits & $0.25 \%$ & $9.85 \%$ & $89.69 \%$ & $0.21 \%$ \\
12000 bits & $0.26 \%$ & $9.32 \%$ & $90.32 \%$ & $0.10 \%$ \\
\hline
\end{tabular}

Fig. 8 summarizes the percentage of time 5.5Mbps and $11 \mathrm{Mbps}$ data rates are chosen as the optimal rate for 90 one second snapshots with the packet size 8000bits. As we observed before, most often the minimum of rates is selected and the high bias towards $11 \mathrm{Mbps}$ is the result of Min and Max rates being equal to $11 \mathrm{Mbps}$.

\section{CONClusion AND Future WORK}

In a wireless network, coded packets can be broadcast with different transmission rates to improve coding gain. Even in a network with a fixed bit-rate, the choice of the rate can affect coding opportunities. Motivated by these observations, in this paper, we investigated the impact of bit-rate selection on network coding in wireless mesh networks. We defined a new metric, ECT, that measures the total time needed by a node to deliver two packets to their receivers given the bitrate for transmitting coded packets. We compared the optimal 



Fig. 7. The ETT/ECT values using different transmission strategies vs. optimal rate

rate (with the least ECT) against two intuitive strategies (Min, Max) for selecting rates. Our evaluations have shown that, 11 Mbps is the best default fixed rate for MIT Roofnet and 5.5 Mbps is usually the optimal rate to transmit coded packets when the individual rate for each receiver is different.

Our future work will focus on developing a practical rate selection scheme for network coding. Since the performance of rate selection associated with network coding depends on which nodes are the intended receivers, coding packets appropriately might improve the performance and we intend to explore this aspect.

\section{REFERENCES}

[1] R. Ahlswede, N. Cai, S. R. Li, and R. W. Yeung. Network information flow. Trans. Information Theory, 46(4):1204-1216, July 2000.

[2] J. C. Bicket. Bit-rate selection in wireless networks. 2005. http://dspace.mit.edu/handle/1721.1/34116.

[3] S. Chachulski, M. Jennings, S. Katti, and D. Katabi. Trading structure for randomness in wireless opportunistic routing. In ACM SIGCOMM, Kyoto, Japan, 2007.

[4] D. D. Couto, D. Aguayo, J. Bicket, and R. Morris. A High-Throughput Path Metric for Multi-Hop Wireless Routing. In Proc. ACM Mobicom, 2003.

[5] G. Holland, N. H. Vaidya, and P. Bahl. A rate-adaptive MAC protocol for multi-hop wireless networks. In Mobile Computing and Networking, pages 236-251, 2001. citeseer.ist.psu.edu/holland01 rateadaptive.html.

[6] A. Kamerman and L. Monteban. Wavelan(c)-ii: a high-performance wireless lan for the unlicensed band. Bell Labs Technical Journal, 2(3):118-133, 1997. http://dx.doi.org/10.1002/bltj.2069.

[7] S. Katti, S. Gollakota, and D. Katabi. Embracing wireless interference: Analog network coding. In ACM SIGCOMM, 2007, 2007.

[8] S. Katti, H. Rahul, W. Hu, M. Medard, and J. Crowcroft. XORs in the air: Practical wireless network coding. In In Proceedings of SIGCOMM, 2006.

[9] M. Lacage, M. H. Manshaei, and T. Turletti. Ieee 802.11 rate adaptation: A practical approach. citeseer.ist.psu.edu/719742.html.

[10] MIT Roofnet. http://www.pdos.lcs.mit.edu/roofnet/.

[11] B. Ni, N. Santhapuri, Z. Zhong, and S. Nelakuditi. Routing with opportunistically coded exchanges in wireless mesh networks. In WiMesh 2006. 2nd IEEE Workshop on Wireless Mesh Networks, 2006, 2006.

[12] S. Sengupta, S. Rayanchu, and S. Banerjee. An analysis of wireless network coding for unicast sessions: The case for coding-aware routing. In INFOCOM 2007. 26th IEEE International Conference on Computer Communications. IEEE, 2007.

[13] S. Zhang, S. C. Liew, and P. P. Lam. Hot topic: Physical-layer network coding. In Proc. ACM Mobicom, 2006. 